Informationsflüsse integrieren

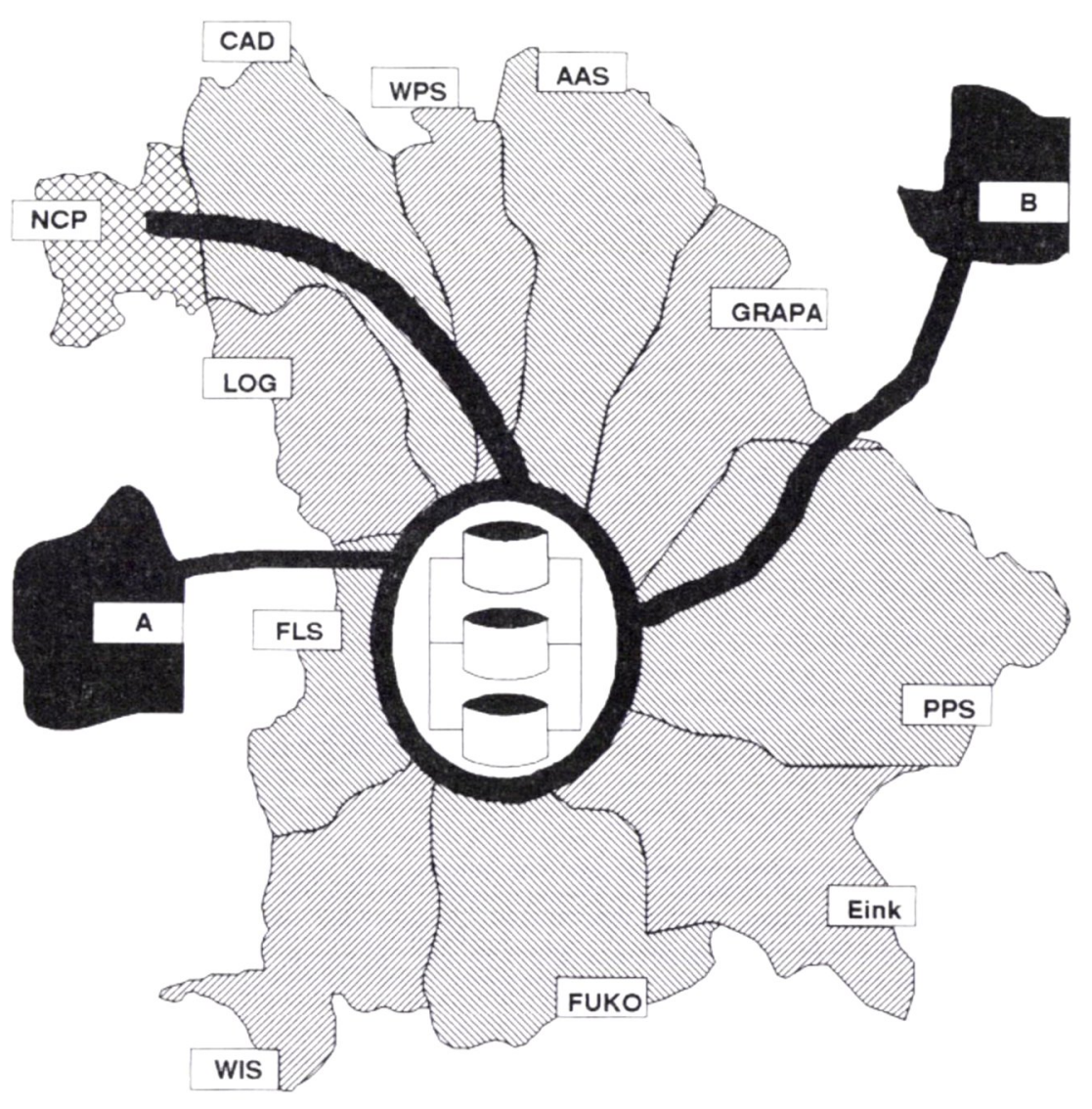

Die Wirtschaftlichkeitsbetrachtung von EDV-Projekten ist ein Balanceakt zwischen herkömmlicher Kosten-NutzenRechnung und strategischer Managemententscheidung. Bei allen Schwierigkeiten der Bewertung gibt es zur Integration der Informationsflüsse im Unternehmen keine Alternative. S. 593

\section{Kooperative Fertigungssteuerung}

Konkurrenz um Ressourcen ist ein zentrales Thema der Fertigungssteuerung. Für eine effiziente Steuerung muß die Kooperation zwischen den dezentralen Bereichen organisatorisch gefördert und informationstechnisch durch gekoppelte Leitstände unterstützt werden.

\section{Statistik und Dokumentation im PPS-System}

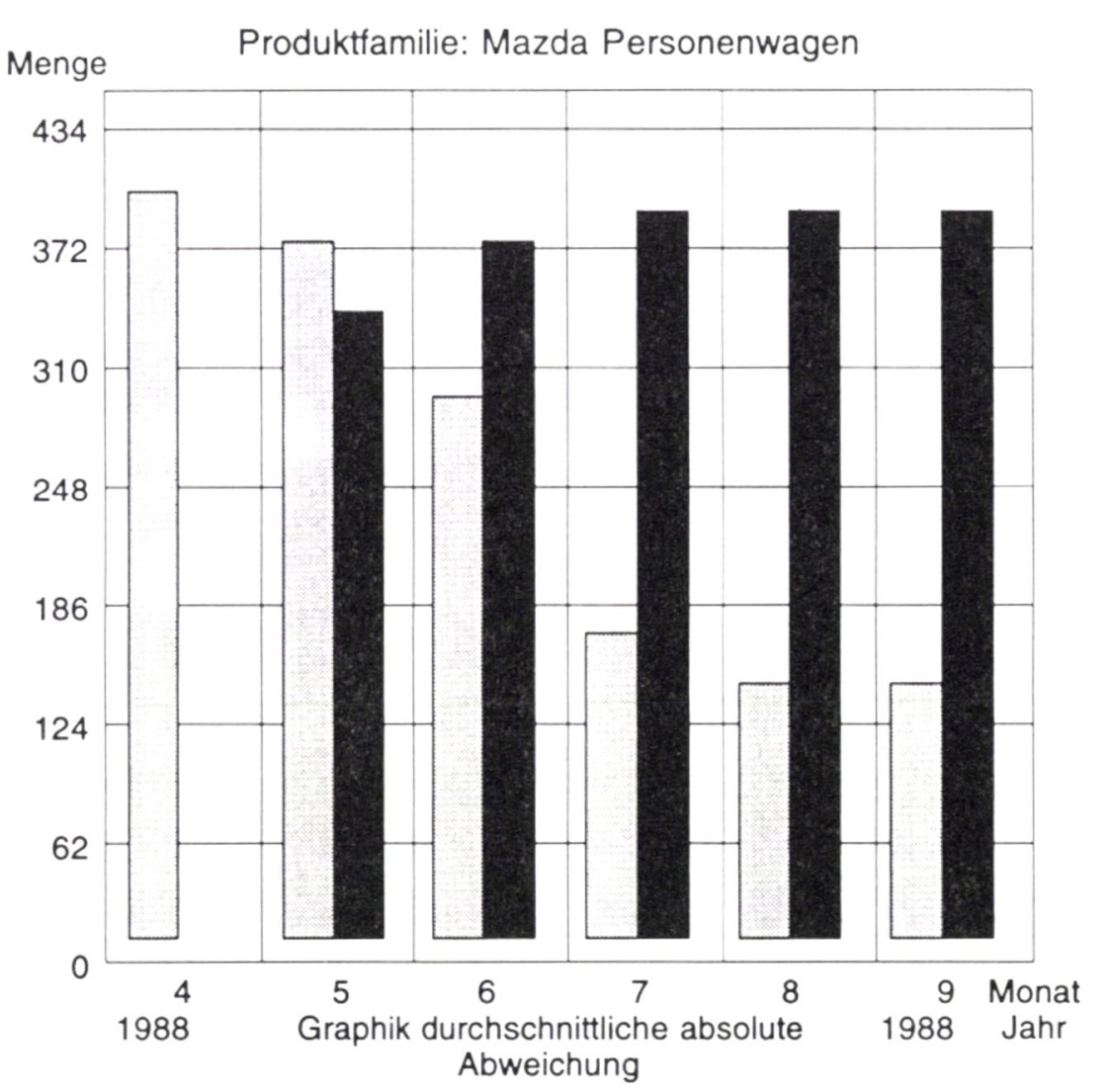

PPS-Systeme müssen über leistungsfähige Funktionen für Statistik und Dokumentation verfügen. Hauptziele dieser Funktionen sind es, die Voraussetzungen für ein umfangreiches Management-Informationssystem zu schaffen und die Erfüllung der Dokumentationspflicht im Rahmen der erweiterten Produzentenhaftung sicherzustellen.

\section{Editorial}

Graphische Bedienungsoberflächen müssen die Software-Ergonomie verbessern

\section{CIM - Rechnerintegrierter Fabrikbetrieb}

G. Spur, D. Specht, M. Meyer: Software-ergonomische Schnittstelle für Ingenieurdatenbanken

Betriebliche Informationen im PPS-System

Mittelständischer Dichtungshersteller als MRPII-Anwender erster Klasse ausgezeichnet

H. Stapfer: Informationsmanagement in der Fertigung

Rechneranwendung in Fertigung und Montage

T. Brinkop, E. Nullmeier: Konkurrenz und Kooperation - zwei Schlüsselkonzepte der Fertigungssteuerung Technologiereport analysiert EDM-Systeme

L. Friedrich, G. Bock, H. Mushack, H. Zakrzewski: Bestands geregeltes PPS-System für kontinuierliche Prozesse

A. Decker: Statistik und Dokumentation - unverzichtbare Bestandteile der Produktionsplanung und -steuerung

PPS-System für den auftragsbezogenen Einzelfertiger

J. Milberg, H. ZetImayer: Intelligente Störungskompensation mit regelnden Produktionsleitsystemen

Unix-System für die betriebliche Datenverarbeitung erweitert

K. Kurbel, C. Rautenstrauch: Graphisches Navigieren durch eine PPS-Datenbasis mit Browsern.

D. Hartkopf: Dokumentenverwaltung und -archivierung als Bestandteil eines integrierten Softwaresystems.

PPS als Instrument des innerbetrieblichen Kostenmanagements

Rechnerunterstützte Konstruktion und Planung

M. Keller, U. Kürsten, T. Kempf-Fischer: Konstruktionsabläufe durch Einsatz einer Ingenieurdatenbank automatisieren

Fabrikanlagen und Materialfluß

J. Schwarz: Durchlaufzeiten in der Werkstattfertigung

H. Stierle: Fahrerlose Transportfahrzeuge ohne Leitlinien - eine neue Dimension im Materialfluß.

Flexibler Materialtransport für die Montage.

Spanen und Gewinderollen in einer Aufspannung

Sperrige Werkstücke auf Senkrechtbearbeitungszentrum fräsen

Hauptzeitparalleles Spannen in einem Bearbeitungszentrum

637

CNC-Bandsägeanlage in Kompaktbauweise.

Notizen

zu Veranstaltungen

Report

CAD $\cdot$ CAM $\cdot$ CIM

Fabrikanlagen und Materialfluß

Impressum 


\begin{tabular}{|c|c|}
\hline Sonderteil: $C A D \cdot C A M \cdot C I M$ & CA 257-CA 320 \\
\hline CAD-Nachrichten der GI & CA 260 \\
\hline Notizen. & CA 263 \\
\hline $\begin{array}{l}\ldots \text { aus der Branche } \ldots \ldots \ldots \\
\ldots \text { aus der Technik. . . . . . . . }\end{array}$ & $\begin{array}{l}\text { CA } 263 \\
\text { CA } 263\end{array}$ \\
\hline
\end{tabular}

G. Spur: Rechnerunterstützung für kreatives und effizientes Konstruieren.

CA 264

\section{Maschinen- und Anlagenbau}

T. Jasper: Ausbau eines CAD-Systems zu einem integrierten Gesamtsystem

A. Kratzer, W. Wallmeier-Wendt: CAD beschleunigt Elektrikund Hydraulik-Konstruktion.

E. Binder: CAD-Modellplanung im Papiermaschinenbau.

Feinwerktechnik und Gerätebau

O. Müller, H. Maier: Von CAD zu CIM

H. Rothenhöfer: Auch Meßmaschinen vom Bildschirm

CA 280

H. Rothenhofer: Auch Meßmaschinen vom Bildschirm

CA 285

CA 291

Werkzeug- und Formenbau

U. Sendler, H. Altmeyer: Gießerei auf dem Weg zur CIM-Lösung CA 296 J. Rößle, S. Schneider: CAD/CAM-Kopplung im Stanzwerkzeugbau

CA 300

Kunststoffverarbeitung

J. Rothley: Rechner verkürzt Durchlaufzeit beim Spritzgießen

CA 304

Qualitätssicherung

W. Eversheim, P. Zeller, B. Kloten: Integration der Prüfplanerstellung in CAD-Systeme

CA 310

Kurzberichte

Mehrkörpersimulation auf dem Personal Computer

CA 269

Integrierte Zeichnungsverwaltung in neuer Version

CA 274

BEM - eine Alternative zur FEM-Technik.

CA 278

Workstation oder RISC-PC?

CA 288

Industrie-PC / Dreidimensionales Entwickeln auf dem PC

CA 290

Photorealistische Bilder mit neuem Schattierprogramm

CA 299

Report

CA 316

Inserentenverzeichnis

CA 318

\section{Regelnde Produktionsleitsysteme}

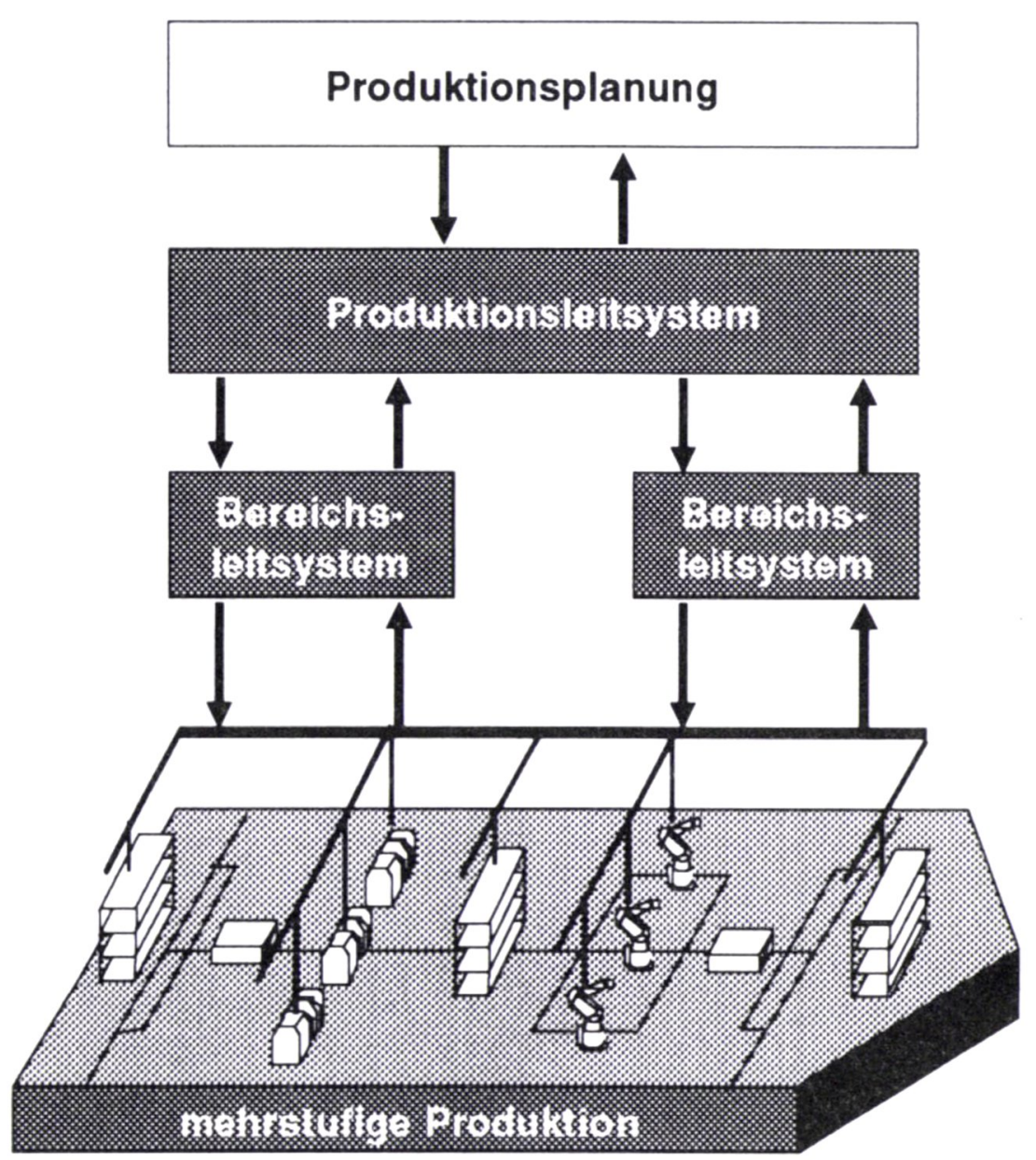

Mit einem entscheidungsunterstützenden Regelansatz und einem entsprechenden Leitstandskonzept kann in absehbarer Zeit den gestiegenen Anforderung an das Störungsmanagement begegnet werden. Erst zusammen mit den Erfahrungen aus der entscheidungsunterstützten Produktionsregelung kann es gelingen, selbständig regelnde Auftrags- und Produktionsleitsysteme zu entwickeln.

\section{Dokumente verwalten und archivieren}

Die Bedeutung von Dokumentenverwaltungssystemen steigt mit den zunehmenden Datenmengen. Integrierte Systeme eröffnen durch die Bereitstellung von graphischen und alphanumerischen Daten an beliebigen Arbeitsplätzen im Unternehmen neue Perspektiven.

S. 621

\section{Sonderteil: $C A D / C A M / C I M$}

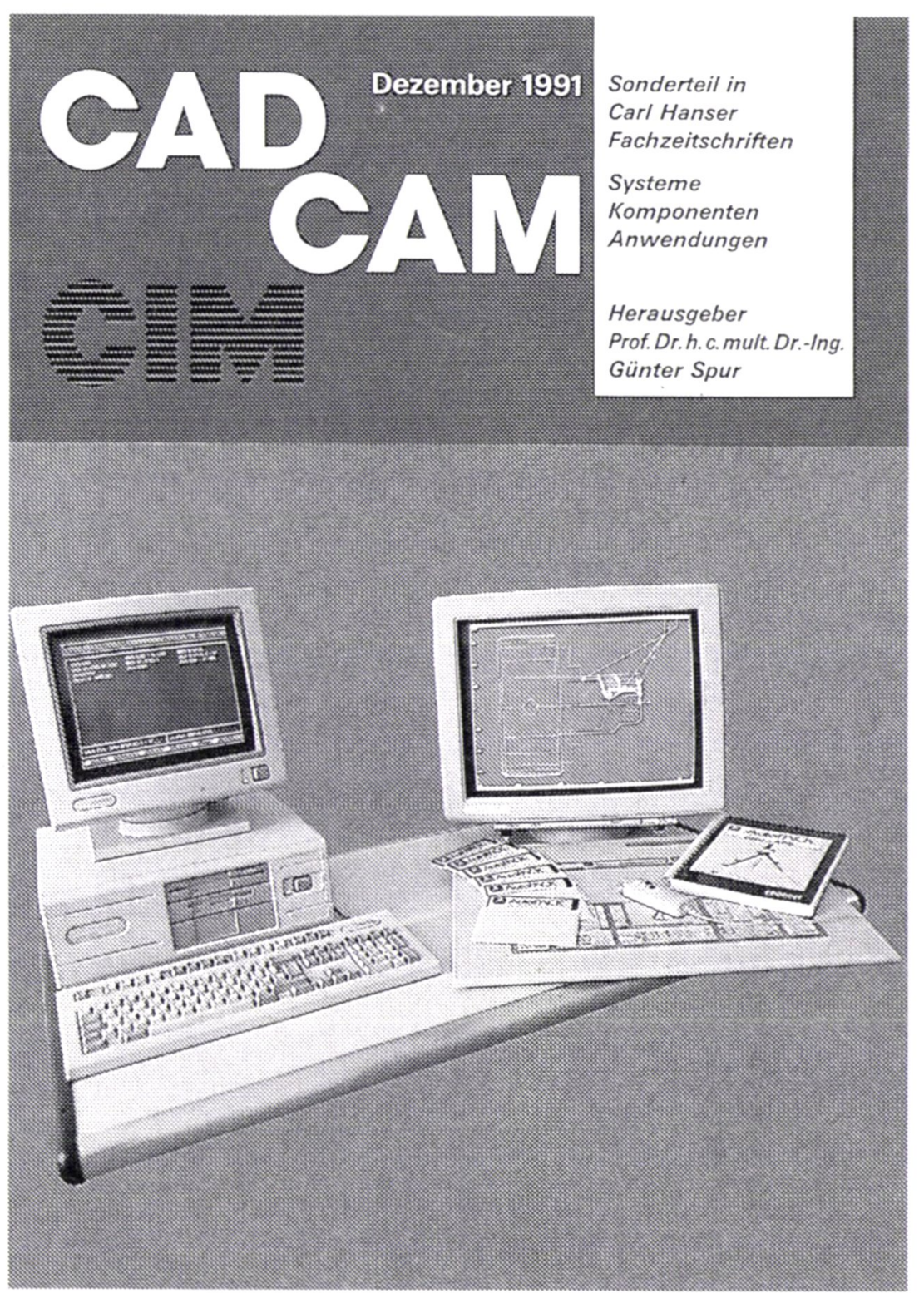

